\title{
El movimiento estudiantil chileno durante la Transición a la Democracia: resurgimiento y movilización contra las herencias de la dictadura ${ }^{1}$
}

\author{
Javiera Errázuriz Tagle ${ }^{2}$
}

Recibido: 6 de marzo de 2017 / Aceptado: 27 de octubre de 2017

Resumen. Este artículo tiene por objetivo analizar la trayectoria del movimiento estudiantil chileno, y en particular de la Federación de Estudiantes de la Universidad de Chile (FECH), en los años 90 para observar las transformaciones que vive el movimiento durante la transición a la democracia. La progresiva desmovilización social impulsada por los partidos de oposición en los primeros años de la transición a la democracia, afectaron a todos los movimientos sociales que habían protagonizado las protestas entre 1983 y 1986, y en particular al movimiento estudiantil, el cual se vio obligado a crear nuevos marcos para la acción colectiva adecuados a la nueva situación de democracia.

Palabras clave: movimiento estudiantil; transición a la democracia en Chile; marcos para la acción colectiva; movilizaciones sociales en el Chile de los años 90.

\section{[en] The chilean student movement during the democratic transition: resur- gence and mobilization against the legacies of the Pinochet regime}

\begin{abstract}
This research paper aims to analyze the path followed by the Chilean student movement, particularly the Federación de Estudiantes de la Universidad de Chile (FECH) in the 90's, in order to evaluate transformations experienced by this movement during the transition to democracy. The ongoing social demobilization, impelled by opposition parties when facing the 1988 referendum, affected all social movements which had led protests between 1983 and 1986, especially the student movement. The mobilizations reappearance during the cycle 1996 - 1997 responded to a profound internal transformation of the student movement patterns and, moreover, to the development of a new framework for collective action suitable for democracy.
\end{abstract}

Keywords: student movement; transition to democracy in Chile; framework for collective action; social mobilization in Chile during the 90's.

Sumario. Introducción. 1. El plebiscito de 1988 y primeros años 90: la Universidad "amarrada". 2. La FECH, ¿para qué?. 3. 1997: "Empresarios, magnates, banqueros / Cruje entera la Universidad". 4. ¿Victoria o derrota?: balance del ciclo de movilizaciones de 1997. 5. Referencias bibliográficas. 6 . Fuentes.

Cómo citar: Errázuriz Tagle, J. (2018): “El movimiento estudiantil chileno durante la Transición a la Democracia: resurgimiento y movilización contra las herencias de la dictadura". Cuadernos de Historia Contemporánea, 40, 349-370.

1 Este texto forma parte de mi Tesis de Doctorado, cuyo tutor fue Manuel Pérez Ledesma, recientemente fallecido. Quisiera dedicar este artículo a Manolo, como una manera de agradecer todo lo que aprendí de él.

2 Universidad Andrés Bello. (Santiago de Chile, Chile). Departamento de Humanidades email: javiera.errazuriz@unab.cl 


\section{Introducción}

Los movimientos sociales tuvieron un papel importante en la recuperación de la democracia en Chile, articulando una plataforma social de oposición al régimen. El movimiento obrero y el estudiantil, unidos a diversos movimientos sociales populares como los movimientos poblacionales, los de asociaciones de víctimas de la represión, los de mujeres, etcétera, movilizaron a cientos de miles de personas en la época de las protestas (1983-1986), en momentos en que los partidos políticos no tenían la capacidad para lanzar una estrategia de movilización social de esta envergadura. Sin embargo, una vez que los partidos reaparecen en el juego político y más tarde son legalizados, asumen el protagonismo de la oposición al régimen.

De acuerdo con diversos autores, la estrategia de movilización social fue fundamental para articular la oposición a la dictadura, y permitió mostrar que una parte importante de la ciudadanía apoyaba un cambio en el sistema político. De este modo, las protestas nacionales contribuyeron al proceso de redemocratización, sin embargo no lograron su cometido de derribar al régimen, y, a partir de 1986, la oposición democrática opta por una salida institucional, que implicó la desmovilización de los movimientos sociales y populares, y la negociación con el régimen. De esta manera, como señala Mario Garcés:

se transitó, en verdad, hacia una democracia elitista -como reconstrucción de un sistema político- inhibiéndose una vez más el desarrollo de la participación ciudadana y de los movimientos sociales. ${ }^{3}$

\section{Según Manuel Antonio Garretón,}

las negociaciones y concertaciones en el nivel de las cúpulas y de las elites tienden a reemplazar las movilizaciones sociales durante la transición democrática y los procesos de consolidación. ${ }^{4}$

La desmovilización producida durante la transición a la democracia en Chile está estrechamente relacionada con dos elementos: la estructura de oportunidades políticas y el pacto entre elites. Las oportunidades políticas, en palabras de Sidney Tarrow, corresponden a dimensiones del entorno político que ofrecen ciertos incentivos para que las personas participen en acciones colectivas. ${ }^{5}$ En el contexto de un gobierno autoritario, las oportunidades políticas son escasas y las restricciones múltiples, por lo que la posibilidad de fracaso es alta en la medida en que el régimen puede reprimir cualquier acción colectiva aun utilizando métodos que están fuera de la legalidad. Sin embargo, llama la atención que, en los años 80 , aun encontrándose bajo un gobierno dictatorial y represivo, los movimientos sociales en Chile iniciaron un extenso ciclo de protesta que, como ya hemos señalado, sirvió para articular la oposición social al régimen. Y, por el contrario, a partir de la transición, los movi-

3 Garcés, Mario: “Los movimientos sociales populares en el siglo XX: balance y perspectivas", Política, Santiago de Chile, primavera, $n^{\circ} 043$, (2004), p. 29.

4 Garretón, Manuel Antonio: "Movimientos sociales y procesos de democratización. Un marco analítico". EXCERPTA, Santiago de Chile, No2, abril (1996), p. 6-7.

5 Sidney Tarrow: El poder en movimiento. Los movimientos sociales, la acción colectiva y la politica, Alianza, Madrid, 2004, p. 116. 
mientos sociales tendieron a reducir drásticamente su nivel de movilización, pese a que muchas de las restricciones que existían bajo Pinochet habían desaparecido. Patricia Hipsher señala que este fenómeno de desmovilización durante los procesos de transición a la democracia-que no es exclusivo del caso chileno- está estrechamente relacionado con el pacto entre elites que posibilita el traspaso de poder desde el régimen autoritario al democrático, por restringido que este último sea. El temor a la involución autoritaria, presente en todos los procesos transicionales, y la necesidad de consolidar la democracia contribuyeron también a la desmovilización social. Las elites gobernantes favorecen, entonces, formas institucionalizadas de participación política que tienden a la no confrontación ${ }^{6}$.

De esta forma, a partir de 1990, y durante los dos primeros gobiernos de la Concertación de Partidos por la Democracia (1990-1999) se privilegia la gobernabilidad, la consolidación democrática y la estabilidad económica, dejando de lado la participación y movilización social, con lo cual, los movimientos sociales entran en períodos de desactivación y, en algunos casos, de desarticulación. ${ }^{7}$

El caso del movimiento estudiantil obedece a la misma lógica señalada por los autores antes mencionados, ya que luego de las fortísimas manifestaciones de 1987, que lograron derribar al rector delegado de la Universidad de Chile, José Luis Federici, el movimiento entra en un período de reflujo bastante profundo, que incluirá la desaparición -por algunos años- de las federaciones estudiantiles más emblemáticas, como la Federación de estudiantes de la Universidad de Chile (FECH) y la Federación de estudiantes de la Universidad de Santiago de Chile (FEUSACH). En este artículo exploraremos las causas de este período de reflujo, pero también analizaremos el resurgir del movimiento estudiantil en 1997 y la articulación de nuevos marcos para la acción colectiva, que critican y problematizan el proceso de transición a la democracia y los gobiernos que lo han llevado a cabo. Los marcos para la acción colectiva son los significados compartidos, los conceptos por medio de los cuales la gente define su contexto. "Resulta imprescindible que las personas, como mínimo, se sientan agraviadas por una situación determinada y crean que la acción colectiva puede contribuir a solucionar esta situación" ${ }^{8}$. Estos marcos mezclan el aspecto simbólico con los principios orientados a la acción, creando así una suerte de mapa que permite convertir la pasividad en acción, y generar un sentimiento de identificación con aquellas personas a las que se quiere movilizar.

Las reivindicaciones estudiantiles de 1997 diferían bastante de las de 1986 o 1987. Ya no existía la dictadura, por ello las manifestaciones callejeras y la ocupación de los espacios públicos no se saldaban con la represión con que lo hacían antes, la acción policial tendía -aunque no siempre- a ajustarse a los procedimientos legales, por lo que el movimiento estudiantil necesitaba, para salir de la etapa de reflujo, un nuevo conjunto de reivindicaciones que le permitiera generar identificación entre los estudiantes y recuperar un espacio significativo en la sociedad civil.

A modo de contexto, cabe señalar que el movimiento estudiantil español tuvo una trayectoria similar en cuanto a desmovilización durante la transición a la democracia.

6 Hipsher, Patricia: "Democratization ant the Decline of Urban Social Movements in Chile and Spain", Comparative Politics, vol. 28, 3 (1996), p. 292

7 Garretón: "Movimientos sociales y procesos..., p. 7.

8 Mc Adam, Doug; Mc Carthy, John y Zald, Mayer: Movimientos sociales: perspectivas comparadas. Madrid, Istmo, 1999, p. 26. 
Si bien el curso 75-76 fue intensísimo en cuanto a movilizaciones ${ }^{9}$, estas decayeron rápidamente en los cursos posteriores. Esto, porque las organizaciones estudiantiles quedaron muy diezmadas ya que los partidos políticos con más presencia en la universidad habían decidido jugar sus cartas en las elecciones y en el Parlamento, dejando así a la deriva a un movimiento que había sido punta de lanza en la lucha contra el franquismo. Asimismo, el fin del franquismo y de la lucha anti-represiva había dejado al movimiento estudiantil sin objetivos, sin propuestas, y prácticamente sin nada qué decir. La revista Cuadernos para el Diálogo señalaba en marzo de 1978:

El movimiento estudiantil se ha desinflado hasta casi desaparecer. Esos miles de estudiantes que durante años llevaron a cabo duras luchas contra la represión del franquismo y contra la política universitaria de las autoridades solo tienen ahora una muy escasa sucesión. ${ }^{10}$

Los pocos estudiantes que participaban en partidos políticos reconocían la dificultad del momento y el descenso dramático de militantes en la universidad, especialmente en los partidos de izquierda: "Un afiliado del PCE reconoce que sus militantes han descendido en un año de cien a quince en la Facultad de CC.PP."11. Por su parte, un estudiante señalaba a Cuadernos que "militar en la Universidad, [era] peor que escuchar ocho horas las palabras de Monseñor Balaguer". ${ }^{12}$

Sin embargo, un nuevo ciclo de protestas comenzó en el curso 79-80, al calor de la discusión sobre el proyecto de Ley de Autonomía Universitaria (LAU), que, según los estudiantes, profundizaba la selectividad en la universidad por motivos económicos. Así, un nuevo conjunto de reivindicaciones, relacionadas con la defensa de una universidad democrática, pública y sin discriminación económica, con autonomía en su gestión, en la configuración de los órganos de gobierno y en la selección del profesorado, articuló el nuevo ciclo movilizador. El 12 de diciembre, más de cuatro mil estudiantes celebraron una asamblea de distrito en la UAM, y se sucedieron diversos encierros y huelgas de hambre, no sólo de estudiantes universitarios sino también de enseñanza media. ${ }^{13}$ Las movilizaciones fueron en aumento, así como los enfrentamientos con la policía (que se saldaron con las muertes de los estudiantes José Luis Montañéz y Emilio Martínez, en diciembre de 1979) y con grupos de extrema derecha (como el Batallón Vasco Español que asesinó a la estudiante Yolanda González en febrero de 1980).

El resurgimiento del movimiento estudiantil estuvo encabezado por estudiantes del Partido de los Trabajadores, organización de izquierda radical que surge en 1979 de la fusión del PTE y CRT. Este partido no tenía representación parlamentaria, y, por lo tanto, no tenía participación política institucional a nivel estatal ${ }^{14}$, por lo que consideraba indispensable tener una presencia lo más amplia posible en los movimientos sociales, de ahí su participación en el movimiento estudiantil y sus críticas

9 Para más detalles sobre este curso ver Errázuriz, Javiera: "El movimiento estudiantil madrileño en los inicios de la Transición a la Democracia: auge y agotamiento de un actor fundamental en la lucha contra el franquismo", Revista Ayer, 99, 3 (2015).

10 Cuadernos para el Diálogo, núm. 253, 4-10 de Marzo de 1978, p. 26. BNE.

11 Cuadernos para el Diálogo, núm. 253, 4-10 de Marzo de 1978, p. 27. BNE.

12 Cuadernos para el Diálogo, núm. 253, 4-10 de Marzo de 1978, p. 30. BNE.

13 El País, 12 de Diciembre de 1979. Hemeroteca digital El País.

14 PTE y ORT tenían concejales en varios ayuntamientos, por lo que su participación local era mayor. 
a los partidos de izquierda como el PCE y el PSOE, que decidieron "abandonarlo" para dedicarse a la política institucional. Finalmente, el ciclo de movilizaciones decayó a partir de marzo de 1980, cuando se entrampa el debate sobre la $\mathrm{LAU}^{15}$, pero fue una movilización importante ya que permitió sacar la discusión de las Cortes y trasladarla a la calle, a las aulas y a los campus universitarios. De este modo, la articulación de nuevos marcos de significado y el liderazgo de grupos de izquierda extraparlamentaria permitieron quebrar el periodo de reflujo del movimiento estudiantil español, y situarlo en un nuevo contexto: el escenario democrático.

Creemos que esta trayectoria es replicable, con las particularidades propias del caso, para el movimiento estudiantil chileno del período de la transición. De este modo, partimos de la hipótesis de que la estrecha vinculación existente entre los partidos de gobierno y los grupos políticos que lideraban las federaciones estudiantiles, particularmente la FECH, contribuyó a la desmovilización del movimiento estudiantil y a su virtual desaparición como actor social relevante durante los primeros gobiernos de la transición chilena. Esta situación solo se superará cuando líderes de izquierda extraparlamentaria, específicamente del Partido Comunista, asuman la dirección de las federaciones y construyan nuevos marcos para la acción colectiva del movimiento estudiantil.

\section{El plebiscito de 1988 y primeros años 90: la Universidad "amarrada"}

Después de tantos años de lucha contra la dictadura, de impulsar movilizaciones sociales, de buscar solidaridad internacional, la oposición decidió enfrentarse al régimen por la vía institucional y participar del plebiscito de 1988, aun asumiendo el riesgo de que los resultados de éste pudieran ser manipulados o desconocidos por Pinochet.

En esta coyuntura, a comienzos de ese año, las movilizaciones sociales tendieron a descender mientras diversos grupos de oposición organizaban la Concertación de Partidos por el $\mathrm{NO}^{16}$. Este conglomerado inició rápidamente una campaña que llamaba a la población a inscribirse en los registros electorales y a votar el 5 de octubre de 1988. ${ }^{17}$ Cerca del $92 \%$ de la población concurrió a inscribirse. El Partido Comunista y alguna facción socialista se negaron inicialmente a participar en el plebiscito, aunque finalmente el PC terminó uniéndose al llamado a votar NO.

El movimiento estudiantil participó con entusiasmo en el plebiscito. Como señala Víctor Muñoz Tamayo, la renuncia del rector delegado Federici en 1987, forzada por el levantamiento de la comunidad universitaria, se entendió como una primera derrota del régimen militar; la segunda -y definitiva derrota- vendría de la mano

15 Finalmente, la LAU no fue aprobada y en 1983, con el PSOE ya en el poder, se redactó y aprobó la Ley de Reforma Universitaria, cuyo título primero otorgaba a la universidad la categoría de servicio público, una reivindicación largamente esperada por los estudiantes.

16 Este conglomerado agrupaba a la Democracia Cristiana, el MAPU, el Partido Humanista, la Izquierda Cristiana, el Partido Verde, el Partido Radical, distintas facciones del Partido Socialista (Almeyda y Núñez) y el Partido por la Democracia.

17 Los registros electorales chilenos fueron destruidos después del golpe de estado de 1973, por lo que había que construir nuevos registros. La inscripción de las personas en dichos registros era fundamental para garantizar la transparencia del proceso. Otros plebiscitos se habían llevado a cabo durante el régimen militar sin la existencia de registros, lo que permitió la manipulación de resultados. 
del triunfo del NO. ${ }^{18}$ Por esta razón, la movilización estudiantil, que había llegado a niveles sin precedentes en 1987, descendió visiblemente en 1988. Además, los partidos de la Concertación necesitaban a sus cuadros juveniles para organizar la campaña y para promover la participación electoral. Los estudiantes comunistas no se plegaron a esta iniciativa y continuaron con el llamado a derrotar a la dictadura a través de la movilización, sin embargo, la mayor parte de los estudiantes de base orientó sus esfuerzos a la votación, en lugar de continuar con una lucha que parecía no tener opciones de triunfar en el corto plazo $^{19}$.

Tras el triunfo del NO y del candidato de la oposición en las elecciones de 1989, y antes de dejar el poder, Pinochet se preocupó de promulgar una serie de leyes que consolidaban el sistema económico neoliberal y hacían más difícil reformar el sistema político. Entre ellas estaba la Ley Orgánica Constitucional de Educación (LOCE), promulgada el 10 de marzo de 1990, apenas un día antes del traspaso de mando. De esta manera, el régimen intentaba asegurarse que la educación -tanto superior como básica y media- siguiera los parámetros fijados en los 17 años de dictadura.

En su artículo $2^{\circ}$, la LOCE señalaba que "La educación es un derecho de todas las personas. Corresponde, preferentemente, a los padres de familia el derecho y el deber de educar a sus hijos; al Estado, el deber de otorgar especial protección al ejercicio de este derecho; $y$, en general, a la comunidad, el deber de contribuir al desarrollo y perfeccionamiento de la educación". ${ }^{20}$ Básicamente, el Estado ya no tenía el deber de proporcionar la enseñanza sino simplemente de proteger ese derecho, traspasando la responsabilidad a los padres y a la comunidad. La LOCE recogía el espíritu del principio de subsidiariedad del Estado con respecto a la educación, y, aunque se promulgó en último momento, esta Ley rigió los destinos de la educación en Chile hasta 2009, cuando fue derogada por la Ley General de Educación. Tal como señala Fabio Moraga, "la salida de los militares no implicó que se desmantelara la institucionalidad autoritaria y contraria al movimiento estudiantil que había caracterizado al régimen de Pinochet", ${ }^{21}$

La LOCE venía a complementar la reforma que el régimen había iniciado en las universidades estatales con la Ley General de Universidades de 1981, que había transformado completamente el sistema de financiamiento de estas y además había excluido explícitamente a los estudiantes de las instancias de gestión y de la elección de autoridades de las universidades.

En este contexto de desmovilización - producto de la adopción de la vía institucional a la democracia- y de nueva legislación, el movimiento estudiantil chileno entró en una clara fase de reflujo a partir de 1990. Esto se debía fundamentalmente a dos motivos. Por un lado, a la expectativa generada en la población por el primer gobierno democrático en 17 años y al deseo de los partidos de la Concertación y de

18 Muñoz Tamayo, Víctor: Generaciones. Juventud universitaria e izquierdas políticas en Chile y México (Universidad de Chile-UNAM 1984-2006), Santiago de Chile, LOM, 2001, p. 119.

19 Thielemann, Luis: La anomalía social de la transición. Movimiento estudiantil e izquierda universitaria en el Chile de los noventa (1987-2000), Santiago de Chile, Tiempo Robado, 2016, p.69.

20 Ley Orgánica Constitucional de Enseñanza, 10 de marzo de 1990. http://www.uchile.cl/portal/presentacion/ normativa-y-reglamentos/8386/ley-organica-constitucional-de-ensenanza.

21 Moraga Valle, Fabio: "Crisis y recomposición del movimiento estudiantil chileno (1990-2000)", en Renate Marsiske (coord.): Movimientos Estudiantiles en la historia de América Latina, México, vol. III. UNAM (2006), p. 185. 
sus seguidores de llevar adelante con éxito y en paz la transición a la democracia. Es importante recordar que, pese a que Pinochet había salido del gobierno, no había desaparecido de la escena política. Seguía "custodiando su obra" desde la Comandancia en Jefe del Ejército, lo que condicionó fuertemente la capacidad de acción del gobierno de Aylwin. Por otro lado, pese a las movilizaciones llevadas a cabo por los estudiantes desde 1984 en adelante, la institucionalidad y la legislación que regía a las universidades era la misma que había dictado el régimen, por lo tanto, salvo algunos cambios específicos (como la elección del rector por parte de los académicos) y victorias simbólicas (como la salida del rector delegado Federici), los estudiantes no habían conseguido reformas en cuanto al financiamiento ni en cuanto a su participación en las instancias de decisión.

Durante 1990 y 1991, la directiva de la FECH estuvo en manos de estudiantes de la Democracia Cristiana Universitaria, que actuaban en consonancia con las directrices de su partido, que gobernaba el país en ese momento. A partir de 1990, la FECH comenzó a recibir financiamiento de parte de la Universidad de Chile, lo que le permitió organizar mejor su labor. Además, el presidente de la federación fue integrado con derecho a voz (no a voto) en las sesiones del Consejo Universitario ${ }^{22}$, de modo que los estudiantes quedaron parcialmente representados en la máxima instancia deliberativa de la Universidad de Chile. Sin embargo, en este período la FECH no hizo mucho más que organizar grandes fiestas y eventos masivos en lugar de mantener su espacio como actor social crítico, debido a que

Las sucesivas directivas FECH (...) no se abocaron temprana ni robustamente a la revisión crítica de las políticas educacionales dejadas por Pinochet, ni al necesario proceso de redemocratización al interior de la universidad. ${ }^{23}$

1992 fue una pequeña excepción en esta etapa de reflujo, ya que en ese año varias federaciones de universidades estatales e incluso privadas fueron ganadas por estudiantes socialistas. Ese año se produjo la Primera Jornada Nacional de Movilización por el Financiamiento Universitario, que buscaba presionar al gobierno para que reformara el sistema de créditos universitarios que impedía el acceso o la continuidad de la educación superior a muchos jóvenes. Ahora bien, a esta jornada no se plegó la FECH (que en ese momento era dirigida por Arturo Barros, de las juventudes del Partido Socialista, mismo partido que el Ministro de Educación de la época, Ricardo Lagos) aunque sí participaron estudiantes de la Universidad de Chile, en su mayoría militantes de la izquierda extraparlamentaria, que encontraron en la universidad un espacio adecuado para desarrollar sus demandas, que casi no tenían cabida en el nuevo sistema político fuertemente constreñido por el sistema electoral binominal.

Los resultados de este mini ciclo de movilización fueron exiguos. El gobierno cambió el sistema de créditos universitarios por el del Fondo Solidario de Crédito Universitario, que buscaba favorecer a los estudiantes de más bajos recursos (pertenecientes al $80 \%$ de la población más vulnerable) para ingresar a las universidades del Consejo de Rectores, pero que en la práctica permitía que las universidades (que

22 Instancia ejecutiva que reúne al Rector y a los decanos en donde se discuten y aprueban las políticas y el presupuesto de la Universidad de Chile.

23 Roco Fossa, Rodrigo: "La FECH de fines de los 90: relatos de una Historia Presente", Anales de la Universidad de Chile, Santiago de Chile, Sexta serie, $\mathrm{n}^{\circ} 17,(2005)$, p. 53-54. 
eran quienes otorgaban los créditos), vendieran la "cartera de deudores" a bancos públicos o privados. ${ }^{24}$ Además, el Fondo Solidario financiaba entre el $50 \%$ y $100 \%$ del arancel de referencia, pero los aranceles reales que cobraban las universidades solían ser bastante más altos. Por otra parte, el crédito se otorgaba con un interés del $2 \%$ y quien recibiera el beneficio debía comenzar a pagar dos años después de haber egresado de la universidad, y la deuda debía estar cancelada en un plazo máximo de 15 años.

Ahora bien, pese a que el Fondo Solidario representaba un pequeño avance con respecto al sistema anterior, no era lo que los estudiantes de izquierda exigían, porque el Estado seguía sin hacerse cargo del financiamiento de la educación superior pública, dejando éste en manos de instituciones bancarias públicas o privadas que manejaban estos créditos como un negocio más. De esta manera, como resultado de la frustración generada por el poco éxito de la movilización, el movimiento estudiantil y las federaciones entraron en una profunda crisis. Esta fue particularmente fuerte en el caso de la Universidad de Chile. En las elecciones de 1993 no se reunió el quórum necesario, por lo que no hubo directiva FECH para 1994 y 1995.

\section{La FECH, ¿para qué?}

Según Rodrigo Roco, presidente de la FECH en 1996 y 1997, lo que gatilló la crisis de las federaciones en general, y de la FECH en particular, fue el progresivo alejamiento entre la directiva y las bases. ${ }^{25}$ La federación de los primeros años 90 estuvo dirigida por militantes de partidos de gobierno (tanto de la DC como del PS), por lo que en este período las demandas estudiantiles estuvieron supeditadas a las estrategias de los partidos políticos en el poder, anulando así la autonomía del movimiento estudiantil. Esta etapa se caracterizó por la desmovilización y "la excesiva instrumentalización de las necesidades y reivindicaciones de los estudiantes que se usaban para hacer política hacia el Estado" ${ }^{26}$. Los mismos dirigentes estudiantiles estaban más preocupados de la política interna de sus partidos que de las reivindicaciones y necesidades de los estudiantes. Según señala Luis Thielemann, "las dirigencias estudiantiles, copadas por militantes de la Concertación, intentaron contener cualquier movilización que pudiese afectar al Gobierno, bajo el argumento de que aquello era "hacerle olitas al gobierno" y con ello provocar un hipotético retorno de los militares"27. Por ejemplo, en este período, se vendió el Canal de TV de la Universidad de Chile, y se impuso el cobro de aranceles a través de letras bancarias, sin que los estudiantes pudieran impedirlo.

Esto se tradujo en el fuerte descenso de la participación de los estudiantes en las elecciones de federación, dando muestras del profundo descrédito en el cual había caído la política universitaria. Sin embargo, los estudiantes más movilizados lograron trasladar su confianza desde los desacreditados militantes de partidos oficialistas a grupos de la izquierda extraparlamentaria, fundamentalmente de las juventudes

\footnotetext{
Moraga Valle, Fabio: Crisis y recomposición... p. 196.

Roco Fossa, Rodrigo: La FECH de fines... p. 54.

Moraga Valle, Fabio: Crisis y recomposición... p. 197-198.

27 Thielemann, Luis: "Para una periodificación del movimiento estudiantil de la transición (1987-2011)". Pretérito Imperfecto, 2011. http://movimientoestudiantil.cl/wp-content/uploads/2015/12/119-Para-unaPeriodificacion-del-Movimiento-Estudiantil-de-La-Transicion-Luis-Thielemann.pdf, p.4.
} 
del Partido Comunista, que parecían no estar "contaminados" ni constreñidos por la dinámica de los consensos. En el caso puntual de la Universidad de Chile, ante la desaparición de la FECH, los presidentes de los Centros de Alumnos se hicieron cargo del movimiento estudiantil, aunque fue muy poco lo que lograron hacer. Hubo que esperar hasta 1995 para que se iniciara un nuevo ciclo de movilizaciones y una nueva reconstitución de la Federación de Estudiantes de la Universidad de Chile.

Después de casi tres años sin directiva, a mediados de 1995 comenzó un complejo proceso de reconstitución de la FECH. Para lograrlo, era urgente re-encantar a la masa estudiantil con un proyecto político adecuado a la época que se estaba viviendo. Los estatutos de la federación databan de 1984 y se habían redactado pensando en la lucha contra el régimen, por eso, en 1995 ya no se correspondían con la realidad. Además, como señala Víctor Muñoz, en los años sin FECH emergieron una serie de demandas propiamente universitarias pero que cuestionaban directamente el proceso de transición vivido en el país. Estas reivindicaciones esbozaban una crítica al sistema neoliberal de mercado, impuesto en dictadura pero gestionado por los gobiernos de la Concertación, y particularmente se dirigían "contra un orden institucional que a nivel nacional conservaba la Constitución de 1980, del mismo modo que, a nivel universitario, lo hacía con los estatutos dictatoriales". ${ }^{28}$ En este contexto, se comenzaba a perfilar unos nuevos marcos para la acción colectiva centrados en la idea de participación de los estudiantes en la toma de decisiones en la universidad. El objetivo era construir alguna forma de gobierno que incluyera a los estudiantes y les permitiera incidir en el gobierno universitario. No era un objetivo sencillo, ya que el fantasma del cogobierno ${ }^{29}$, que desde la reforma de 1967 rondaba la universidad, fue utilizado con fuerza por quienes se oponían a la participación estudiantil.

En octubre de 1995 se realizaron nuevas elecciones de la federación, con estatutos provisionales y el compromiso de redactar estatutos definitivos, en cuya confección debían participar estudiantes de todas las tendencias. En estas elecciones salió elegido presidente Rodrigo Roco, militante de las Juventudes Comunistas y líder del colectivo de Estudiantes de Izquierda (EEII) con un 36,8 \% de los votos. En segundo lugar, quedó la Juventud Socialista con un 29,4 \%. En tercer lugar, la DCU con un $20,4 \%$ y, finalmente, la derecha con un $13,3 \%$. De esta manera y según el nuevo sistema proporcional, la FECH de 1996, de carácter transitorio y refundacional ${ }^{30}$ quedó conformada por un comunista en la presidencia, un socialista en la vicepresidencia, un democratacristiano en la secretaría general y dos independientes de izquierda en la secretaría de comunicaciones y en la secretaría de finanzas.

A comienzos de 1996, la nueva directiva FECH comenzó a rearticular el movimiento estudiantil y a intentar frenar el proceso de desmantelamiento de la Universidad de Chile. Así, por ejemplo, ante la noticia de que el Rector licitaría la administración de la Radio de la Universidad de Chile a privados, un grupo de estudiantes liderados por la FECH decidió ocupar pacíficamente las oficinas de la radioemisora. Aunque pueda parecer un detalle, la situación de la Radio reflejaba bastante bien el

28 Muñoz Tamayo, Víctor. Generaciones... p. 122.

29 Por cogobierno se entendía la participación de académicos, estudiantes y funcionarios en la elección de las autoridades universitarias. La Reforma Universitaria de 1967 había conseguido esto, y a partir de 1968, la elección del Rector y los Decanos se hacía a través del claustro pleno (tri-estamental, aunque con diferentes porcentajes por estamento), pero esta conquista fue eliminada luego del golpe de 1973 y los estudiantes quedaron fuera de cualquier instancia de decisión de las políticas universitarias.

30 Roco Fossa, Rodrigo: La FECH de fines de los 90... p. 55 
panorama de la Universidad de Chile en general. En un comunicado dirigido a la comunidad universitaria y nacional, la FECH señalaba que:

el grave deterioro de la Radioemisora y sus dificultades financieras no son más que el resultado de la nefasta política de autofinanciamiento universitario que fue impuesta en nuestro país a principios de la década de los 80 . A ello se suma la escasa preocupación de las actuales autoridades por esta área de nuestro quehacer como Casa de Estudios. El silencio que se le impone a la Universidad al despojarla del último de los medios con los que ella se relaciona masivamente con la sociedad, contrasta con la ausencia de democracia y participación a la hora de llevar a cabo estas medidas que involucran su esencia misma. ${ }^{31}$

De esta manera, la nueva directiva de la FECH comenzó su estrategia movilizadora a partir de los problemas puntuales de la Universidad de Chile, para luego abrirse a los problemas que afectaban a todo el sistema público de educación superior:

Ya no queremos ser mudos testigos del desmantelamiento de esta Universidad, la que ha servido y debe seguir al servicio de los intereses superiores del desarrollo social, democrático, cultural y económico de nuestro país. ${ }^{32}$

En este sentido, la nueva FECH llevó adelante dos procesos fundamentales para la reconstitución del movimiento estudiantil: la redacción de nuevos estatutos y el Congreso Constituyente, que se celebró en septiembre de 1996. Este Congreso fue una instancia esencial en el proceso de reconstitución y de reconducción de la federación, ya que en él se redactó una Declaración de Principios, en la cual se establecieron las prioridades y proyectos a los que se dedicaría la $\mathrm{FECH}$, así como nuevas formas de gobierno de la federación. En primer lugar, la Declaración de Principios señalaba que la federación era la máxima instancia de representación de los estudiantes de la Universidad de Chile. Básicamente, la FECH se planteaba como una instancia de los estudiantes, independiente del gobierno y de la autoridad académica, que contenía en su seno a todas las corrientes democráticas que quisieran participar en la federación, y que tenía como misión la lucha por un sistema económico y político:

Auténticamente democrático, basado en la igualdad de oportunidades, la solidaridad, la participación, la libertad y la justicia, que garantice a todos los chilenos una vida digna, vivienda, trabajo, educación, salud, recreación, cultura... ${ }^{33}$

Respecto de la forma de gobierno, se estableció que la FECH tuviera mayor diversidad política lo que logró a través de un sistema proporcional para elegir a la directiva, y a través de los consejeros, que eran representantes de las diversas facultades, y que se elegían de acuerdo con el número de estudiantes de cada una.

De esta manera, la FECH de 1996 logró articular un conjunto de reivindicaciones y demandas, entre las que se encontraban la defensa de la educación pública, el

31 “A la comunidad universitaria y nacional", FECH, Santiago, 11 de marzo de 1996, p. 1. Archivo FECH.

32 "A la comunidad universitaria y nacional", FECH, Santiago, 11 de marzo de 1996, p. 1. Archivo FECH.

33 Declaración de principios de la Federación de Estudiantes de la Universidad de Chile, Santiago, 8 de septiembre de 1996 citado en Moraga Valle, Fabio: Crisis y recomposición... p. 200. 
problema del autofinanciamiento y del déficit estructural en los presupuestos de la Universidad de Chile, y la falta de espacios de participación estudiantil en la toma de decisiones de la universidad, que permitieron reconstruir el movimiento estudiantil y recobrar la legitimidad perdida en los primeros años de la transición.

En orden a relanzar la movilización, la FECH denunció la crisis por la que atravesaba la educación superior, cuyo origen se situaba en la ilegitimidad de la institucionalidad y la legislación impuesta por el régimen militar-particularmente de la LOCE- pero también se culpaba a los gobiernos de la Concertación de no haber hecho gran cosa por cambiar la situación. En 1992, el gobierno de Patricio Aylwin había presentado al Congreso el proyecto de Ley Marco de las Universidades Estatales General de Educación Superior, que intentaba modificar la legalidad heredada de la dictadura, reformando los Estatutos de las Universidades Estatales, pero no tocaba la exclusión de los estudiantes de la participación en el gobierno de la universidad ni el sistema de financiamiento. Además, el proyecto de ley otorgaba una fuerte influencia del poder ejecutivo en el gobierno de las universidades estatales, sin un aumento del aporte fiscal directo a estas. ${ }^{34}$

Según un documento de la FECH, publicado en 1996, dicho proyecto únicamente buscaba mecanismos legales que hicieran más eficiente la gestión universitaria, para poder competir en igualdad de condiciones con el creciente número de universidades privadas. Esto contrastaba con el descenso en el aporte que el Estado entregaba a la educación superior (Universidades, Centros de Formación Técnica e Institutos Profesionales) el cual, según el presupuesto de 1996, era de un 0,67 \% del PIB. Esta imposición de criterios de mercado estaba afectando a los estudiantes de diversas maneras. La más directa era la que decía relación con la capacidad económica del estudiante o de su familia, ya que la posibilidad de acceder y de mantenerse en la universidad dependía exclusivamente de eso.

Ello se refleja en que la Universidad es pagada, y cada año suben los aranceles, los cuales están en más o menos 2.000 dólares promedio (se le considera un bien de consumo por parte del gobierno), se reducen las posibilidades de acceder a beneficios bajo el eufemismo de "focalizarlos" en los más pobres, los que, en las condiciones existentes en la sociedad chilena, "no llegan" a la universidad. ${ }^{35}$

Otro ámbito afectado era la calidad de la enseñanza misma. Los estudiantes consideraban que la docencia impartida en las universidades no tenía un verdadero compromiso con la realidad del país porque se orientaba a las necesidades del modelo neoliberal. Lo más grave, según el documento era que esta noción de educación pública atravesada por el modelo neoliberal había generado:

Una pérdida creciente del sentido nacional, de responsabilidad y aporte al desarrollo nacional, de combate de las injusticias y de esfuerzos frente a los problemas nacionales (desigualdad creciente, pérdida y destrucción de la identidad nacional y latinoamericana, superación de la pobreza, destrucción del medio ambiente, etc.). ${ }^{36}$

34 Muñoz Tamayo, Víctor: Generaciones. Juventud Generaciones... p. 120.

35 Rodrigo Roco; a los compañeros de la UNEB. Santiago, 1996, p. 1. Archivo FECH.

36 Rodrigo Roco; a los compañeros de la UNEB. Santiago, 1996, p. 1. Archivo FECH. 
Una vez diagnosticada la crisis de la educación superior chilena, la FECH señalaba las formas de enfrentarla, para lo cual proponía la articulación de un movimiento estudiantil concienciado, orientado a reforzar la relación entre sociedad y universidad, y, sobre todo, comprometido con la democratización del gobierno universitario.

En suma, la nueva federación tenía dos grandes banderas de lucha: la crisis del sistema público de educación superior, generada por la legalidad e institucionalidad impuesta por el régimen militar y mantenida por los gobiernos de la Concertación (en este ámbito, los principales temas eran el autofinanciamiento y la falta de participación de los estudiantes en los procesos de toma de decisión), y la articulación de un gran movimiento social que defendiera la noción de educación pública, que estaba siendo destruida por la aplicación de los criterios del libre mercado. Estas dos grandes reivindicaciones constituyeron los pilares de los nuevos marcos para la acción colectiva en democracia, que se plasmaron en el ciclo de movilizaciones de 1996-1997, el momento de mayor conflictividad estudiantil en lo que iba de la transición a la democracia.

\section{1997: "Empresarios, magnates, banqueros / Cruje entera la Universidad"37}

El conflicto de 1997 marca un hito en la movilización estudiantil chilena de los años 90 porque se hace cargo del conjunto de aspiraciones y reivindicaciones de los estudiantes desde los años 80 en adelante. Aunque ya no se luchaba contra la dictadura porque se había producido el tránsito hacia la democracia, las demandas del movimiento estudiantil de los 90 estaban en sintonía con las de la década anterior: cambio en el sistema de financiamiento, participación de los estudiantes en el gobierno de la universidad, recuperación de la relación entre universidad y sociedad, en el entendido de que el país requería de un sistema de educación pública (escolar y superior) que no estuviera dominado por criterios de mercado.

En este sentido, la reconstitución de la FECH en 1995 y el posterior Congreso Constituyente de 1996 funcionaron como una oportunidad política, una instancia impulsora de lo que posteriormente será el conflicto estudiantil más complejo de la década de los 90. A partir de marzo de 1997, la mesa directiva de la FECH comenzó a trabajar en un petitorio que resumía las demandas de los estudiantes de la Universidad de Chile. Este documento fue aprobado en el Pleno de la federación ${ }^{38}$ por una amplia mayoría, y fue presentado al Rector Jaime Lavados el 19 de mayo. Los puntos más importantes del petitorio aprobado por la FECH decían relación con la renuncia del Rector y la realización de un proceso amplio de discusión-que incluyera a toda la comunidad académica- sobre los Estatutos de la Universidad de Chile y el Plan de Desarrollo Estratégico. ${ }^{39}$

Junto a esto, la federación convocó a una jornada nacional de movilización para exigir el cambio en el sistema de financiamiento de la educación superior pública y

37 Dos primeros versos del himno reestructurado de la Universidad de Chile compuesto por un académico destacado, citado en Roco Fossa, Rodrigo: La FECH de fines de los 90... p. 66.

38 Instancia en la que se reúne la directiva con el Cuerpo de Consejeros y el Consejo de Presidentes de Centros de Alumnos.

39 Roco Fossa, Rodrigo: La FECH de fines de los 90... p. 67. 
para manifestarse en contra de la Ley Marco que, desde 1993, dormía en el Congreso. Así, a través de la combinación de reivindicaciones propias para la Universidad de Chile y otras de nivel nacional, el movimiento estudiantil consiguió salir de su letargo e iniciar un nuevo ciclo de movilizaciones que no sólo cuestionaban al sistema educativo del país sino también al proceso mismo de transición a la democracia, desde el momento en que éste estaba operando bajo una institucionalidad heredada de la dictadura, y, por lo tanto, ilegítima para una parte importante de la población. Apenas una semana antes, el rector Lavados aseguraba a El Mercurio que no existían incendios en la "U" $"$, y que los problemas que había estaban identificados y en vías de solución. Ante la pregunta por la participación de los académicos en las instancias de gobierno universitario, señaló que:

En una universidad los profesores no participan de grandes y vociferantes asambleas. Ahora, cuando hacen presentaciones por escrito y utilizan las instancias reglamentarias me parece que participan lo suficiente ${ }^{41}$.

Con el objetivo de atraer el apoyo de la comunidad académica, considerado indispensable para el éxito de la movilización, la FECH publicó una carta abierta a todos los académicos de la universidad, en la cual se los invitaba a participar del proceso de reforma que esperaban realizar. Los estudiantes partían su análisis con un diagnóstico crítico de la situación:

En nuestra Universidad existe la más profunda crisis desde el término de la intervención militar. Ella supera, a la vez que abarca, las dificultades y diversos problemas locales por todos conocidos. Se trata de una crisis global, que involucra el sentido, la vida cotidiana y la proyección de nuestra Universidad. ${ }^{42}$

Culpaban también a la falta de democracia interna que impedía "un abordaje adecuado, diverso y propositivo sobre las variadas dificultades que nos apabullan cotidianamente". ${ }^{43}$ Además, se hacían cargo de la compleja situación que vivían los académicos de la Universidad, los cuales tenían que optar a fondos concursables o a recursos privados para poder realizar sus investigaciones. En la carta abierta a los académicos, la FECH establecía claramente su propuesta a la comunidad universitaria, la cual partía por la renuncia del Rector y seguía con la convocatoria a un Congreso Universitario, con participación de los tres estamentos. La carta finalizaba apelando a la misión de la Universidad de Chile:

Los invitamos a que nos ayuden a defender y a recrear este espacio que es, sobre todo de ustedes. La Universidad que los cobijó y educó, la que en más de alguna oportunidad les ha dado la satisfacción privilegiada de sentirse parte constructora

40 Forma coloquial de referirse a la Universidad de Chile.

41 Rodrigo Barría: “¿Lavado de Manos? Rector de la Universidad de Chile pasa a la ofensiva”. El Mercurio, 11 de mayo de 1997, cuerpo D, p. D6.

42 Carta abierta a los académicos de la Universidad de Chile, FECH, Santiago, 26 de mayo de 1997, p. 1. Archivo FECH.

43 Carta abierta a los académicos de la Universidad de Chile, FECH, Santiago, 26 de mayo de 1997, p. 1. Archivo FECH. 
de un país mejor, o ha permitido el simple anhelo de ampliar los horizontes de las preguntas y respuestas. ${ }^{44}$

El 28 de mayo, tres mil estudiantes de la Universidad de Chile marcharon por las calles de Santiago exigiendo la renuncia del Rector y una respuesta al petitorio. Ante el silencio de las autoridades, el proceso movilizador continuó y se produjeron paros y tomas en 13 facultades, y además se plegaron al movimiento estudiantes de la Universidad de Santiago (USACH), de la Universidad Técnica Metropolitana (UTEM) y del ex Pedagógico ${ }^{45}$. Las críticas al sistema de financiamiento de la educación superior estatal y los problemas de democracia interna de las universidades no eran exclusivos de la Universidad de Chile, de modo que los nuevos marcos para la acción colectiva permitieron generar un movimiento más amplio, que convocó a movilizaciones en distintas universidades y en diferentes regiones del país. La Confederación de Estudiantes de Chile (CONFECH), que durante la decadencia de las federaciones estudiantiles casi había desaparecido, volvió a surgir con fuerza y se dedicó a coordinar las movilizaciones.

Ahora bien, la relación entre estudiantes y académicos, al menos en la Universidad de Chile, no sería tan fluida debido a la exigencia de renuncia del Rector. Jaime Lavados había sido el primer Rector no designado luego de 17 años de régimen militar; lo habían escogido los académicos en 1990 y lo habían reelegido en 1994. Por esto, la petición de renuncia del Rector aparecía ante los ojos de los profesores como un cuestionamiento a su capacidad de decisión, aunque muchos no estaban de acuerdo con la gestión de Lavados.$^{46}$ Es por esto por lo que el Consejo Universitario rechazó completamente la petición de los estudiantes, y por esto también, fue más difícil acercar posiciones entre un estamento y otro. Pese a esto, los estudiantes sabían lo importante que era el apoyo de los académicos a sus demandas, por lo que no insistieron en la petición de renuncia. En este sentido, el movimiento había hecho un aprendizaje político importante del conflicto de 1987, cuando toda la comunidad universitaria se unió en torno a un conjunto de reivindicaciones, y logró sacar a Federici del Rectorado. Por su parte, el Gobierno declinó oficiar de mediador en el conflicto entre los estudiantes y autoridades universitarias del país, pese a la militancia demócratacristiana de Lavados. La FECH también realizó propuestas para debatir los puntos principales del petitorio redactado en mayo. El 1 de junio propuso al Consejo Universitario la celebración de un congreso que discutiera, frente a la comunidad universitaria, las dos reivindicaciones centrales de los estudiantes: la redacción del Estatuto y el Plan de desarrollo, en donde se delinearían las políticas de financiamiento y gestión de la universidad. En paralelo, los académicos de la Facultad de Filosofía emitieron una declaración pública en la que coincidían con el diagnóstico hecho por los estudiantes respecto de la situación de la universidad.

44 Carta abierta a los académicos de la Universidad de Chile, FECH, Santiago, 26 de mayo de 1997, p. 4. Archivo FECH.

45 El Ex Pedagógico es la actual Universidad Metropolitana de Ciencias de la Educación (UMCE). Hasta 1981 formó parte de la Universidad de Chile y para el movimiento estudiantil de los años 80, el regreso del Pedagógico a la Universidad fue una reivindicación permanente. Nunca se consiguió.

46 Moraga Valle, Fabio: Crisis y recomposición... p. 203. 
Reconocemos que el papel histórico de la Universidad de Chile en la sociedad chilena y en la cultura del país se ha visto menoscabado gravemente en los últimos 25 años. Este proceso de deterioro, que debió detenerse y revertirse con el advenimiento de la democracia, continúa hasta hoy. Reconocemos, asimismo, que hay importantes problemas no resueltos al interior de la Universidad, problemas que no han sido abordados adecuadamente por las instancias universitarias que corresponde. Nos referimos al estatuto, a la actualización de un marco de desarrollo consensuado que represente el sentir de la comunidad académica, al rol del Estado frente a la Universidad, al crédito universitario y a la redistribución del presupuesto histórico, entre otros. ${ }^{47}$

Además, convocaron a un claustro al cual invitaron a toda la comunidad universitaria. Por su parte, los académicos de la Facultad de Ciencias también contribuyeron a la coordinación entre estudiantes y profesores a través de una declaración pública en la que compartían el diagnóstico crítico de la situación en la Universidad de Chile.

La acción de los alumnos es consecuencia de la realidad que ellos viven diariamente en la Universidad de Chile de hoy y debe ser apreciada como una manifestación responsable de un sector de la comunidad universitaria legítimamente preocupado por el destino de la Institución. La percepción de crisis por parte de los alumnos es compartida por los académicos de esta Facultad. Recogemos por ello el llamado a reflexionar y reconstruir la Universidad de Chile. ${ }^{48}$

Los académicos de la Facultad de Ciencias criticaban a la administración Lavados por su actuar divorciado de la comunidad universitaria, aunque no solicitaban la salida del Rector. Se iba gestando así la necesaria coordinación entre los estamentos, especialmente entre estudiantes y académicos, en torno a un conjunto de demandas comunes, como, por ejemplo, los problemas de financiamiento, la falta de democracia al interior de la Universidad de Chile y la urgencia de nuevos Estatutos. El Claustro académico de la Facultad de Medicina Norte emitió una declaración pública, con fecha 3 de junio de 1997, en la cual asumía varios de los planteamientos del movimiento estudiantil, y con particular fuerza rechazaba la imposición de los criterios de libre mercado en la educación.

La aplicación de la economía social de mercado al estilo neoliberal monetarista e inmediatista chileno a la educación ha socavado los cimientos de la educación solidaria estatal en Chile. La Universidad de Chile, exponente máximo de esta educación solidaria estatal, es la institución que más ha sufrido por la imposición desde fuera y desde dentro de este modelo económico que, por esta razón, ha visto la destrucción de lo más preciado que tenía: su pluralismo. (...) Las universidades son consideradas como empresas con fines de lucro y se estima que deben ganar dinero a como dé lugar. La Rectoría de la Universidad de Chile suprime los fon-

47 A la opinión pública, Claustro Académico de la Facultad de Filosofía y Humanidades de la Universidad de Chile, Santiago, 28 de mayo de 1997, p. 1. Archivo FECH.

48 Los académicos de la Facultad de Ciencias a la Opinión Pública. Santiago, 1997, p. 1 Archivo FECH. 
dos propios para investigación científica. Se introduce la errónea concepción del alumno como cliente. ${ }^{49}$

Así, la declaración de los profesores de Medicina, y en general las de todos los claustros académicos, deja entrever no sólo un diagnóstico crítico con respecto al sistema de educación superior, sino también un desencanto con la clase política y los gobiernos democráticos, que no habían sido capaces o no habían tenido la voluntad política de modificar la institucionalidad heredada de la dictadura y, de hecho, habían profundizado la crisis. El Ministro de Educación de la época, José Pablo Arellano (militante de la Democracia Cristiana), defendió la labor del gobierno del Presidente Frei señalando que los problemas de la Universidad de Chile no se debían a la falta de financiamiento y que el gobierno no había descuidado a la Universidad.

Creo que el sistema de educación superior, mirado en perspectiva y no en la coyuntura de los últimos días, pasa un momento expectante en cuanto a sus posibilidades. (...) Lo que sucede es que, efectivamente ha existido más atención en el desarrollo de la enseñanza básica y media ${ }^{50}$.

Sin embargo, estas declaraciones fueron rápidamente rebatidas por autoridades de la misma casa de estudios, particularmente por Patricio Basso, Vicerrector de Economía y Administración. Basso, a través de una declaración demoledora, desmintió al Ministro señalado que en los últimos 22 años, los aportes del Estado a la Universidad de Chile habían disminuido drásticamente, y que dicho proceso no se había detenido con la llegada de la democracia. Entre 1974 y 1980, la Universidad de Chile vio descender sus aportes en un 29,4 \% (26.393 millones de pesos de 1996), por lo que la participación de la Universidad de Chile en el financiamiento fiscal a la educación superior bajó de un 51,6 \% en 1974 a un 43,1 \% en 1980. Con la legislación de 1981, el aporte fiscal descendió aún más, y la participación de la universidad en este llegó al 28,8 \%. Lo más grave, en todo caso, es que esta tendencia no se revirtió con los gobiernos democráticos, y en 1996, la participación de la Universidad de Chile en el financiamiento fiscal del sistema universitario cayó al 22,4 \%. Por cierto, Basso señalaba también que el gobierno de Patricio Aylwin había intentado paliar en parte el daño hecho a la universidad a través de aportes complementarios, que lograron aumentar la participación en un par de puntos porcentuales, pero dichos aportes decrecieron durante el gobierno de Frei. ${ }^{51}$ Se abría así un nuevo flanco en el conflicto entre el gobierno, las autoridades universitarias y los estudiantes.

Los estudiantes eran particularmente críticos con la gestión del Rector Lavados (aunque desistieron de la petición de renuncia) y algunos académicos también, sin embargo, la generalidad del estamento docente no estaba por pedir la renuncia de Lavados porque ellos mismos lo habían elegido dos veces. Además, entre los académicos operaban diferencias políticas importantes. Aquellos cercanos a la Democracia Cristiana apoyaban al Rector (militante DC), mientras los profesores de izquierda

49 A la opinión pública, Claustro académico de la Facultad de Medicina Norte de la Universidad de Chile, 3 de junio de 1997, p. 1. Archivo FECH.

50 Rodrigo Barría: "Las Universidades Vacías. Ministro de Educación Analiza el Paro Estudiantil”. El Mercurio, 14 de junio de 1997, cuerpo D, p. D4

51 Declaración Pública, Patricio Basso, Vicerrector de Economía y Administración de la Universidad de Chile. Santiago, 5 de junio de 1997, p. 1-2. Archivo FECH. 
tendían a ser más críticos. Con todo, el principal interpelado en este conflicto era ciertamente el gobierno. Así, el 11 de junio de 1997, la FECH publicó una declaración dirigida a la comunidad universitaria, en la que hacía un llamado a académicos, funcionarios y autoridades de la universidad para que se sumaran a la demanda que los estudiantes pretendían presentar ante el gobierno.

El momento que se ha creado es inédito en relación con la situación de postergación en que hemos estado sumidos por más de dos décadas. Por lo mismo, el llamado de la FECH es a jugárnosla ahora por abrir los espacios necesarios a nivel del país y el Estado, sin descuidar la adecuada e impostergable solución que requiere la inmediata y mediata situación de nuestra Universidad. ${ }^{52}$

Para ello, proponía tres elementos fundamentales: un Congreso Constituyente, triestamental, participativo y resolutivo, el fin de las políticas de autofinanciamiento, y la constitución de una Mesa Nacional para la Reforma de la Educación Superior y Universitaria ${ }^{53} \mathrm{La}$ insistencia de la federación en que el debate y la toma de decisiones incluyeran a los estudiantes generó una sensación de temor entre las autoridades universitarias y políticas. La petición de participación de los estudiantes en 1997 fue vista por las autoridades como un intento de regreso al cogobierno, considerado por los sectores más conservadores como el culpable de la radicalización de las universidades a fines de los años 60 y comienzos de los 70 . Pese a que los mismos estudiantes aclararon que no pretendían el cogobierno, sino que querían democratizar el gobierno universitario, el Rector y el Ministerio pusieron el tema del cogobierno al centro del debate, como una estrategia para alejar a los estudiantes y académicos moderados del conflicto. El Ministro, en entrevista con El Mercurio, recordaba que ya en los años 60 había existido cogobierno, entendido como la participación de los estudiantes en la elección del Rector, política que el gobierno rechazaba tajantemente ${ }^{54}$. Asimismo, las autoridades universitarias culpaban a grupos de la izquierda extraparlamentaria, especialmente el PC, de estar manipulando al movimiento estudiantil y de perseguir motivaciones políticas tendentes a desestabilizar al gobierno. Según el Secretario General de la Universidad de Chile y también militante DC Francisco Cumplido, "no resulta tan descabellado pensar que en un año electoral ${ }^{55}$ el PC esté intentando sacar el máximo provecho a situaciones conflictivas para el Gobierno" 56 .

Para desarticular estas acusaciones, los líderes universitarios adoptaron una postura moderada, señalando que no pedían participar en la elección de las autoridades académicas, simplemente querían estar representados y tener derecho a opinar respecto de las políticas universitarias de corto y largo plazo, por ejemplo, respecto de la elaboración de los nuevos estatutos de la Universidad de Chile, que a la altura de

52 A los estudiantes, a las autoridades, a la comunidad universitaria, FECH, Santiago, 11 de junio de 1997 , p.1. Archivo FECH.

53 A los estudiantes, a las autoridades, a la comunidad universitaria, FECH, Santiago, 11 de junio de 1997, p.1. Archivo FECH.

54 Rodrigo Barría, "Las Universidades Vacías. Ministro de Educación Analiza el Paro Estudiantil”. El Mercurio, 14 de junio de 1997 , cuerpo D, p. D5

55 En diciembre de 1997 se celebraban elecciones parlamentarias en el país.

56 Rodrigo Barría, "El Juicio Final, ¿Los Últimos Días de Lavados en la U. de Chile?”. El Mercurio, 1 de junio de 1997, cuerpo D, p. D1 
junio de 1997 se estaban discutiendo y redactando a puertas cerradas y de espaldas a la comunidad académica.

En vista de la nula respuesta de las autoridades a las peticiones de la federación y de los académicos, el 12 de junio un grupo de estudiantes se tomó la Torre 15, edificio donde funcionan los servicios administrativos de la Universidad, paralizando así toda actividad burocrática. El 13 de junio, el Claustro de académicos de la Facultad de Ciencias Sociales emitió una declaración en la cual coincidían con los estudiantes en el diagnóstico crítico de la situación de la Universidad de Chile y emplazaban a las autoridades universitarias a incorporar a la comunidad en la discusión respecto de los Estatutos, el plan de desarrollo y el modelo de financiamiento.

Exigimos al Consejo Universitario, al Rector y al Decano de nuestra Facultad, dedicar sus esfuerzos a lograr del poder ejecutivo una iniciativa legal sobre educación superior que permita una profunda transformación de nuestra Universidad. Reconocemos la legalidad de nuestras autoridades en razón de haber sido elegidos por la comunidad académica; sin embargo, creemos que ellos deben legitimarse representando efectivamente el pensar y sentir de los académicos que los eligieron. ${ }^{57}$

De esta manera, la dilatación de la solución al conflicto, y especialmente la actitud de Lavados, de hacer oídos sordos ante las peticiones de la comunidad universitaria, acercaron cada vez más a estudiantes y académicos por lo que el Rector fue quedando progresivamente aislado. Especial rechazo provocó en diversos claustros académicos -y por supuesto, entre los estudiantes- el ingreso de la fuerza pública a algunas facultades, con el fin de desalojarlas. Esta actitud de la policía violaba flagrantemente la autonomía universitaria, y aumentaba el rechazo hacia el Rector, toda vez que dicho ingreso había sido solicitado por él.

El 17 de junio, el Consejo universitario dio un paso importante en la solución del conflicto al aprobar la realización de un Congreso tri-estamental para discutir los problemas de la Universidad, lo cual fue celebrado por los estudiantes, aunque persistieron en las movilizaciones. El 19 de junio, se dio otro paso en la solución al conflicto, con la creación de la Comisión de Proyecto Institucional (CPI), que estaría encargada de generar y organizar la discusión tri-estamental para los nuevos Estatutos, el Plan de desarrollo y el sistema de financiamiento. Con todo, y aunque la Comisión representaba un avance, su creación se hizo, una vez más, a puertas cerradas y sin consultar a la comunidad universitaria, lo que provocó una profunda molestia en el movimiento estudiantil. La desconfianza que existía entre los estudiantes y las autoridades universitarias era uno de los principales impedimentos para lograr una solución. Los estudiantes no creían que las autoridades realmente consultaran a la comunidad las decisiones importantes (no lo habían hecho hasta ahora, y no daban muchas señales de querer hacerlo) y acusaban al rector de estar pautado por el llamado Grupo de Amigos del Rector, "una pequeña cofradía integrada por una elite demócratacristiana de no más de cinco funcionarios que se encargan de orientar y

57 "Declaración del Claustro convocado por los propios académicos de la Facultad de Ciencias Sociales de la Universidad de Chile", Santiago, 13 de junio de 1997, p. 1. Archivo FECH. 
presionar al rector para que este actúe de un modo y otro" 58 . Por su parte, las autoridades universitarias, especialmente el Rector, condicionaban la participación de los estudiantes en la toma de decisiones a la deposición de tomas y paros.

El 21 de junio, más de seis mil estudiantes se reunieron en el Estadio Chile (actual Víctor Jara), en donde se convocó a nuevas movilizaciones para presionar al gobierno. En este contexto, el 26 de junio, un grupo de estudiantes intentó ocupar la Casa Central de la Universidad de Chile, pero el Rector ordenó el desalojo, el cual fue filmado por cámaras de televisión y transmitido en todos los noticieros de televisión. Este grado de violencia en la universidad no se veía desde los años del régimen militar, y por supuesto, supuso la quiebra de las conversaciones entre la FECH y las autoridades universitarias.

Sin embargo, había un hecho que preocupaba particularmente a estudiantes, académicos y autoridades: de prolongarse el conflicto más allá de julio, el año académico estaría perdido, por lo que todas las partes involucradas decidieron flexibilizar sus posiciones y reanudar el diálogo. Durante el mes de junio se produjeron dos claustros tri-estamentales, en donde académicos, estudiantes y funcionarios presionaron para que la Comisión de Proyecto Institucional fuera elegida democráticamente y tuviera representación de los tres estamentos ${ }^{59}$ Esto fue finalmente aprobado por el Consejo Universitario, y el 29 de junio el Pleno de la FECH aceptó deponer tomas y paros.

\section{4. ¿Victoria o derrota?: balance del ciclo de movilizaciones de 1997}

Pese al fin de las movilizaciones en la Universidad de Chile, estas continuaban a nivel nacional. El 30 de junio hubo un intento de toma del Ministerio de Educación y el 1 de julio, más de diez mil estudiantes marcharon hacia el Congreso Nacional en Valparaíso, exigiendo una reforma a la educación superior. Por su parte, el gobierno prometió aumentar los aportes fiscales a las universidades públicas y el 7 de julio reenvió al Congreso el proyecto de Ley Marco para las Universidades Estatales, con algunas modificaciones.

El problema era que la Ley Marco no producía transformaciones sustanciales en el sistema de financiamiento, por lo que no contó con apoyos mayoritarios en el Congreso. Tampoco hubo acuerdo entre los parlamentarios para reformar los estatutos de las universidades estatales, por lo que ante la imposibilidad de hacer reformas sustanciales los gobiernos de la concertación sólo realizaron cambios menores. Así, la LOCE, heredada de la dictadura, solo fue derogada en 2009, cuando el gobierno de la socialista Michelle Bachelet promulgó la Ley General de Educación.

En términos concretos, el conflicto de 1997 terminó con una victoria parcial y una derrota. La victoria parcial fue para los estudiantes de la Universidad de Chile, que consiguieron algunos de los puntos del petitorio presentado al Rector Lavados en mayo de ese año. Por ejemplo, consiguieron que la discusión respecto de los nuevos Estatutos de la universidad incorporara a toda la comunidad universitaria; también lograron -apoyados por los académicos y los funcionarios-que la Comisión de Pro-

58 Rodrigo Barría: “El Juicio Final, ¿Los Últimos Días de Lavados en la U. de Chile?”. El Mercurio, 1 de junio de 1997, cuerpo D, p. D17

59 Profesores $60 \%$, alumnos $30 \%$, funcionarios $10 \%$. Moraga Valle, Fabio: Crisis y recomposición... p. 210. Muñoz Tamayo, Víctor: Generaciones... p. 131 
yecto Institucional (CPI) fuera tri-estamental. Dicha comisión discutió por varios meses temas como el sistema de financiamiento, las formas de gobierno interno, y los mecanismos de gestión de la Universidad de Chile. ${ }^{60}$ La CPI fue una instancia clave para resolver el conflicto de 1997 y dar salida a algunas de las peticiones de los estudiantes. Según Rodrigo Roco:

Dicha comisión (...) cumplió a cabalidad su rol como articuladora de un amplio e inédito proceso universitario de discusión. Ella fue sobre todo pionera de la reconstrucción de las confianzas y respetos mutuos que son la base de las nociones de democracia y comunidad universitarias. ${ }^{61}$

Probablemente, su aporte más importante fue la creación de un Senado Universitario, organismo tri-estamental, encargado de aprobar las normas referidas a los Estatutos y a los Planes de Desarrollo. Esta instancia, que existe hasta la actualidad, también debe ratificar el presupuesto anual de la universidad. Para efectos de nuestro trabajo, lo más interesante de este Senado es que contradecía a la LOCE, que, heredada de la dictadura, negaba la participación de los estudiantes en cualquier instancia de gobierno o debate en torno a la universidad.

En suma, el conflicto de 1997 supuso un avance para la situación de la Universidad de Chile, si bien no tanto en cuanto al financiamiento sí en cuanto a la participación de los estudiantes en las instancias de debate de las políticas universitarias. Así, pudieron participar tanto en la redacción de los Estatutos para la Universidad de Chile $^{62}$ como en el Senado Universitario. Además, el conflicto de 1997 reforzó la noción de comunidad universitaria, entendida como un cuerpo de deliberación en donde todos sus miembros tienen derechos y deberes.

Ahora bien, aunque la Universidad de Chile logró sacar ganancias del conflicto de 1997, no ocurrió lo mismo a nivel nacional. En otras universidades los estudiantes no lograron la participación que se había conseguido en la Universidad de Chile, ni tampoco pudieron modificar sustancialmente el sistema de financiamiento. En este sentido, una vez que la FECH depuso las tomas y los paros, a fines de junio de 1997, se produjo un quiebre en la $\mathrm{CONFECH}$, ya que otras federaciones querían continuar la movilización para seguir presionando al gobierno. El problema era que, sin la FECH, la CONFECH perdía una parte sustancial de su fuerza. De esta manera, y pese a que casi todos los años se producían movilizaciones puntuales en torno al financiamiento, estas no alcanzaron la masividad del ciclo de 1997, y, por cierto, no produjeron transformaciones mayores en el sistema de educación superior hasta la derogación de la LOCE en 2009.

Con todo, no podemos reducir el éxito o fracaso del movimiento estudiantil solamente a los cambios producidos o a las reivindicaciones conquistadas. Y en este sentido, pese a que a nivel nacional la movilización de 1997 no consiguió todo lo que se proponía, sí creemos que tuvo éxito en un aspecto fundamental, ya que el movimiento estudiantil puso el acento en las carencias de la transición a la democracia chilena y quebró la dinámica de desmovilización impuesta por el pacto entre

60 Moraga Valle, Fabio Crisis y recomposición... pp. 211-212.

61 Roco Fossa, Rodrigo: La FECH de fines de los 90... p. 77-78.

62 En 2004 se hicieron modificaciones a los Estatutos heredados del régimen militar y en 2006 se aprobaron Estatutos nuevos. 
elites que llevó adelante el proceso transicional. Concordamos con Fabio Moraga, en considerar al conflicto estudiantil de 1997 como el primer impulso democratizador durante la transición:

En un país donde, paradójicamente, la transición a la democracia protagonizada por la clase política, los militares y los empresarios, ha tendido a reforzar los esquemas autoritarios fijados por la Constitución de $1980 .{ }^{63}$

En este contexto, el movimiento estudiantil chileno recuperó una faceta que le era propia, incluso desde antes del régimen militar, de crítica al sistema político y de impulsor de cambios sociales. El conflicto de 1997 dejó en evidencia que el movimiento criticaba no solamente un aspecto puntual del sistema público de educación superior, sino un modelo específico de sociedad

Que ahoga la noción de ciudadanía reemplazándola por la de consumidor, y para el cual la democracia es ineficiente y molesta, y la política no es más que administración. ${ }^{64}$

Así, los estudiantes salieron a las calles a exigir mayor financiamiento para la educación superior, mayor participación en la gestión universitaria, y cambios en la institucionalidad heredada de la dictadura, es decir, se movilizaron por un modelo social más inclusivo y participativo, en suma, más democrático. De esta manera, el movimiento estudiantil adecuó sus marcos para la acción colectiva al contexto democrático, pasando de luchar contra el régimen militar, a luchar contra sus herencias en la educación superior.

\section{Referencias bibliográficas y otras fuentes}

Garcés, Mario: "Los movimientos sociales populares en el siglo XX: balance y perspectivas". Política, primavera, $n^{\circ}$ 043, Universidad de Chile, 2004.

Garretón, Manuel Antonio: "Movimientos sociales y procesos de democratización. Un marco analítico". EXCERPTA 2, 1996.

Moraga Valle, Fabio: "Crisis y recomposición del movimiento estudiantil chileno (1990-2000)". Renate Marsiske (coord.), Movimientos Estudiantiles en la historia de América Latina, vol. III. UNAM, México, 2006.

Hipsher, Patricia: "Democratization ant the Decline of Urban Social Movements in Chile and Spain", Comparative Politics, Vol 28, No. 3 (apr. 1996).

Mc Adam, Doug; Mc Carthy, John; Zald, Mayer: Movimientos sociales: perspectivas comparadas. Madrid, Istmo, 1999.

Muñoz Tamayo, Víctor: Generaciones. Juventud universitaria e izquierdas políticas en Chile y México (Universidad de Chile-UNAM 1984-2006). LOM, Santiago de Chile, 2011.

Roco Fossa, Rodrigo: "La FECH de fines de los 90: relatos de una Historia Presente". Anales de la Universidad de Chile, Sexta serie, 17, Santiago, 2005.

63 Moraga Valle, Fabio: Crisis y recomposición del movimiento estudiantil chileno... p. 249.

64 Roco: "La FECH de fines de los 90", p. 82. 
Tarrow, Sidney: El poder en movimiento. Los movimientos sociales, la acción colectiva y la política, Alianza, Madrid, 2004.

Thielemann, Luis: La anomalía social de la transición. Movimiento estudiantil e izquierda universitaria en el Chile de los noventa (1987-2000), Santiago de Chile, Tiempo Robado, 2016.

Ley Orgánica Constitucional de Enseñanza, 10 de marzo de 1990. http://www.uchile. $\mathrm{cl} /$ portal/presentacion/normativa-y-reglamentos/8386/ley-organica constitucionalde-ensenanza.

\section{$* * * *$}

A la comunidad universitaria y nacional, FECH, Santiago, 11 de marzo de 1996. Archivo FECH.

A los compañeros de la UNEB, Rodrigo Roco, Santiago, 1996. Archivo FECH.

Carta abierta a los académicos de la Universidad de Chile, FECH, Santiago, 26 de mayo de 1997. Archivo FECH.

A la opinión pública, Claustro Académico de la Facultad de Filosofía y Humanidades de la Universidad de Chile, Santiago, 28 de mayo de 1997. Archivo FECH.

Los académicos de la Facultad de Ciencias a la Opinión Pública, Santiago, 1997. Archivo FECH.

A la opinión pública, Claustro académico de la Facultad de Medicina Norte de la Universidad de Chile, 3 de junio de 1997. Archivo FECH.

Declaración Pública, Patricio Basso, Vicerrector de Economía y Administración de la Universidad de Chile. Santiago, 5 de junio de 1997. Archivo FECH.

A los estudiantes, a las autoridades, a la comunidad universitaria, FECH, Santiago, 11 de junio de 1997. Archivo FECH.

Declaración del Claustro autoconvocado por los académicos de la Facultad de Ciencias Sociales de la Universidad de Chile, Santiago, 13 de junio de 1997. Archivo FECH.

Barría, Rodrigo, “El Juicio Final, ¿Los Últimos Días de Lavados en la U. de Chile?”. El Mercurio, 1 de junio de 1997, cuerpo D.

Barría, Rodrigo "Las Universidades Vacías. Ministro de Educación Analiza el Paro Estudiantil". El Mercurio, 14 de junio de 1997, cuerpo D. 